\title{
Colorectal cancer in elderly patients : An epidemiologic study at University Hospital of Tlemcen
}

Type of article: Conference abstract

$\mathrm{N}$ kara zaitri-benmansour, S Ghomari

Department of Medical Oncology at University Hospital center of Tlemcen. benmansouryesmine@yahoo.fr

\begin{abstract}
:
Background: Colorectal cancer has a high incidence and occurs formost patients aged more than 65 years.[1]This population is a very heterogenus group,ranging from the very fit to the very frail.it has often been untreated due to the comorbid diseases,[2.3]however,thanks to comprehensive geriatric assessement which can detect unsuspected health problems,medical care of these patients has been improved[4]

The objective of this study is to establish the epidemiological,clinical ,histological and therapeutic profiles of colorectal cancer in this population

Methods: We carried out a prospective and descriptive study that includes 69 elderly patients with colorectal cancer treated from january 2016 to december 2018 in medical oncology department at the University Hospital Center of Tlemcen

Results: average age at diagnostic is71years[66-92].The comorbid diseases consist in High Blood Pressure :(46\%),diabetes(27\%),other cardiovascular diseases(11.6\%) and dyslipidemia(6\%).Right sided colon was more often achived (57\%) than the left one(11\%) Adenocarcinoma is the most frequent histological type $(59 \%)$. The tumor was classifed as stage I (10\%),stageII(23\%),stageIII(24.6\%) and stageIV(26\%).Lymphnodes metastasis were observed in $18.5 \%$ of cases,metastasis were localised mostly in liver(77\%). 58\% of patients were assigned to conservative surgery wile $7 \%$ inderwent radical one. $26 \%$ of patients had adjuvant chemotherapy, $20 \%$ palliative one associated with target agents and $6 \%$ had concomittent chemoradiotherapy.

The mean geriatric score of G8 was 13,so the type of drugs and doses were adapted according to geriatric evaluation.The toxicity of treatment was dominated by diarrhea grade1 in $10 \%$ of cases ,neuropathies grade 1 in $7 \%$,vomiting grade 3 in $4 \%$ and hand-food syndrom grade 3 in $1.5 \%$

The average survival is 13 months

Conclusion: Aging is one of the factors we need to take into account to establish treatment strategy of elderly with colorectal cancer, however,patients aged more than 65 years must be treated in the same way as younger subjects together with a personalised strategy considering the comorbidities ,performance status and life style
\end{abstract}

Keywords: colorectal cancer,elderly,comorbid disease,geriatric assessment

\section{Conflict of interest statement}

This article is a conference abstract presented at the International Congress on Health Sciences and Medical Technologies, Tlemcen Algeria 5-7 December 2019, ICHSMT'19.

\section{Authors' biography}

No Biography.

\section{References}

No references 